\title{
Study on Prediction Method of Fluvial Facies Sandbody in Fluvial Shallow Water Delta
}

\author{
Wang Lu \\ (Reaearch institute of petroleum exploration \& development, Beijing, China, 100083)
}

\begin{abstract}
Shallow lake basin fluvial-dominated delta front subfacies's sandbody are widely distributed, and the thin sand body, small scale and diversification as the main features, and they are quite different from delta front in the classic delta sedimentary model developed large-scale and thick estuary sand dam sedimentary. At present, most of them in the development of the oil producing degree is poor, is the main target area in Eastern old oil reservoir, fine anatomy and in-depth understanding of shallow lacustrine fluvial-dominated delta front subfacies's single sandbody formation, distribution characteristics and sedimentary model is particularly urgent. Taking Yong 56-96 block in the southern part of Song Fangtun oil field as an example, this paper analyzed and compared several prediction methods of sand bodies, and made joint prediction of seismic and seismic reservoirs in the study area, thus effectively identified thin channel sand bodies and sheeted sand bodies.
\end{abstract}

Keywords: River-dominated delta; Logging curve; Prediction of sand body; Combination of well and seismic

\section{Introduction}

In the history of oil and gas exploitation in the world, the oil and gas development in sandstone reservoir accounts for a great part of the total oil and gas production. With the development of oil and gas exploration in continental basins of China, fine prediction of sand bodies is becoming more and more important ${ }^{[1-2]}$. Shallow lake basin fluvial-dominated delta front subfacies's sandbody are widely distributed, and the thin sand body, small scale and diversification as the main features ${ }^{[3-7]}$, and they are quite different from delta front in the classic delta sedimentary model developed large-scale and thick estuary sand dam sedimentary. At present, most of them in the development of the oil producing degree is poor, is the main target area in Eastern old oil reservoir, fine anatomy and in-depth understanding of shallow lacustrine fluvial-dominated delta front subfacies's single sandbody formation, distribution characteristics and sedimentary model is particularly urgent. Sand body prediction methods include sedimentary facies analysis, seismic attribute analysis, seismic inversion methods and so on. The delta frontal sand and sheet sand are widely developed in the Yong 56-96 block in the southern part of Song Fangtun oil field. The thickness is thin and thin, the interbed develops, and the sand body changes rapidly in the lateral direction.Purpose layer in the study area is putaohua reservoir, the sand body is thin, identify the single sand body is very difficult, the sand bodies change laterally rapidly, simply by logging data is difficult to control the interwell sand distribution, it was decided to carry out well and seismic reservoir prediction in the study area.

\section{Application and effect analysis of seismic attributes}

Application of seismic attribute analysis in reservoir prediction, geological characteristics of the study area must be clear, the existing geological laws should be grasped, guiding the use of actual seismic data and analysis, the solution of the influence of seismic data to a minimum.Sand and mudstone interbed in study area, and the reservoir is thin, most under $3 \mathrm{~m}$, used the spectral decomposition for the target stratum, selected single frequency body that is more sensitive to the goal layer, and the extraction of inter layer attributes was further carried out. The seismic attributes that reflect the seismic response of sand bodies in the target section were optimized by various means

\subsection{Selection of time window}

In view of the interface properties of the permanent 56-96 section, two extraction methods were carried out respectively, which were along the layer and between layers. After analysis and comparison, it was considered that there was no information about the layer attributes reflected by the inter layer attributes, so the method of inter layer attributes extraction was adopted mainly for the target layer. Through the experiment of window expanding up and down, compared and analyzed the attribute maps in different time windows. It was found that the attribute maps extracted from different time windows reflect different sand distribution trend. (1) When we extracted the target section, the distribution trend of sand body was clear. (2) When we extracted the objective layer from top to bottom extension $1 \mathrm{~ms}$, strong reflection reflected sand body assumes the crumb, the shape of river could not be identified, the main reason was the objective layer was thinner, extending the $1 \mathrm{~ms}$ up and down covered too much information in the other layers. (3) When we extracted the object layer up and 
down by $2 \mathrm{~ms}$, it contained more information, reflecting the less desirable effect of the channel sand. Therefore, seismic attributes analysis was mainly carried out by extracting the interlayer attributes for the target layer at present.

\subsection{Spectrum analysis}

In order to better characterize the channel sand of P1 reservoir, we used S- transform method to carry out time-frequency analysis. Compare S-transform with the conventional discrete Fourier transform and the continuous wavelet packet transform, sampling seismic signals used moving windows can generate accurate real time - frequency chart, and the relationship between the spectrum is more strict, and the computing speed is faster. The predicted results of the s-transformation spectrum decomposition P13 reservoir group were shown in figure 1.1.
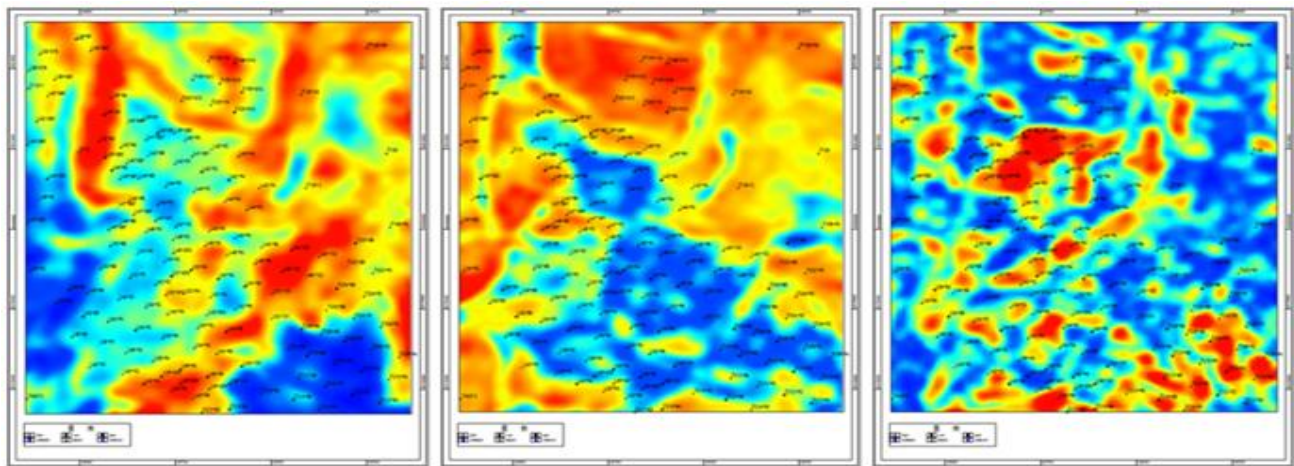

Figure1.1 Frequency plane diagram by S- transform method(10hz、20hz、40hz)

The reservoir sand body was thin and with a thin layer of mudstone, through the spectrum analysis we got seismic data bodies with multiple frequencies, finding the single frequency body which was most suitable for the thickness of the target layer. On this basis, taking the oil layer as the target layer, the attributes were extracted and analyzed, and the plane distribution characteristics of each layer were predicted. Spectral analysis technique can improve the vertical resolution of seismic data and reveal the high-frequency thin layer details which can not be seen by full frequency range data.

\subsection{Optimization of seismic attributes}

The core content of seismic attribute optimization is the use of human experience or mathematical methods, optimal selected combination of seismic attributes or seismic attributes that is most sensitive, effective and representative to the reservoir and the lowest number of attributes. The P122 reservoir group was taken as an example to introduce the optimization process of seismic attributes. A number of seismic attributes were extracted according to the target layer, and the inter layer attributes were extracted based on the physical attributes. Through the intersection analysis, removed attribute's contents repeated and attributes that cannot be interpreted. By comparing and analyzing the distribution characteristics of sand bodies in target stratum, removed attributes that were not associated with the features of the sand body, and were disorganized and irregular in attribute information. After screening and analysis, we got the attributes which could reflect the geological laws better, which could be used to guide the further prediction. Three properties of P122 formation in Putaohua layer were chosen to extract $50 \mathrm{~Hz}$ single frequency body: (1) the arithmetic mean amplitude, (2) the composite absolute amplitude, (3) the root mean square amplitude.From the comparison of sand body development and seismic attribute plan (Figure 1.2) in the target layer, the development of sand body in the single well on the target floor was in good agreement with the seismic attributes.

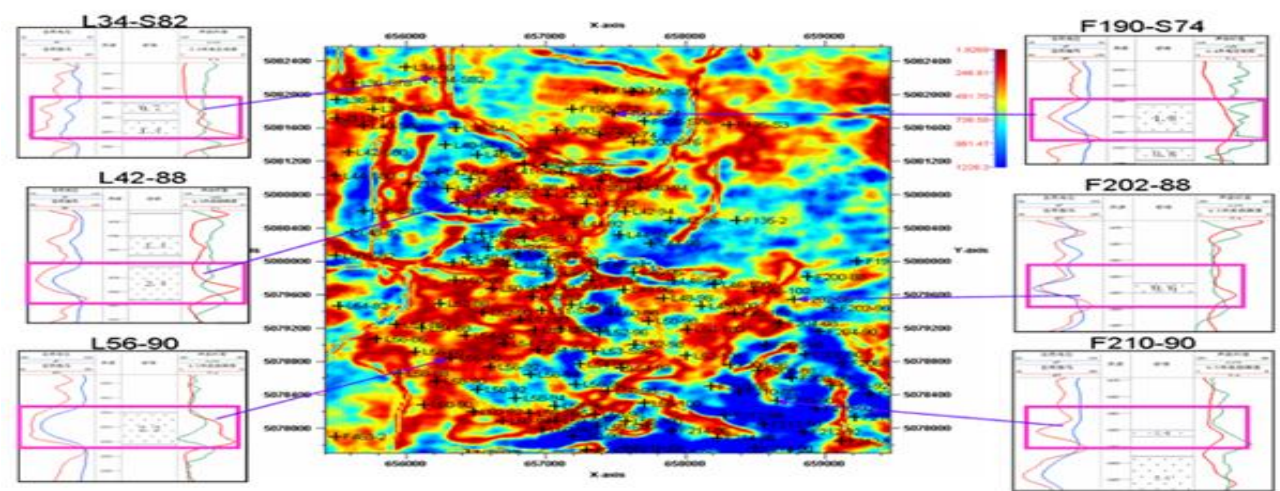

Figure1.2 P122 single well sandbody development and Plane seismic attribute contrast analysis diagram 
From the comparison between distribution of plane sedimentary facies in target layer and Target layer seismic attribute map (Figure 1.3), there were many kinds of sand bodies in the target layer. The purpose of the research area developed various types of sand body, the continuity was stronger, and the sand body was contiguous.
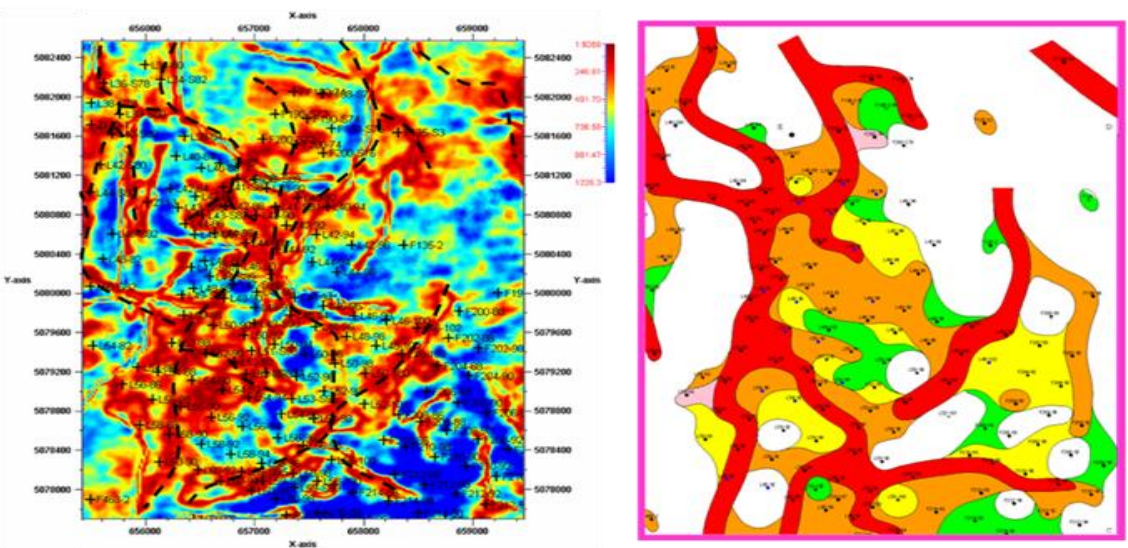

Figure1.3 P122 comparison analysis of plane sedimentary facies and seismic attributes

Seismic attribute extraction was carried out for each target stratum in the study area, the seismic attribute analysis and optimization, which could reflect the characteristics of the reservoir to each plane attribute was shown below (Figure 1.4).
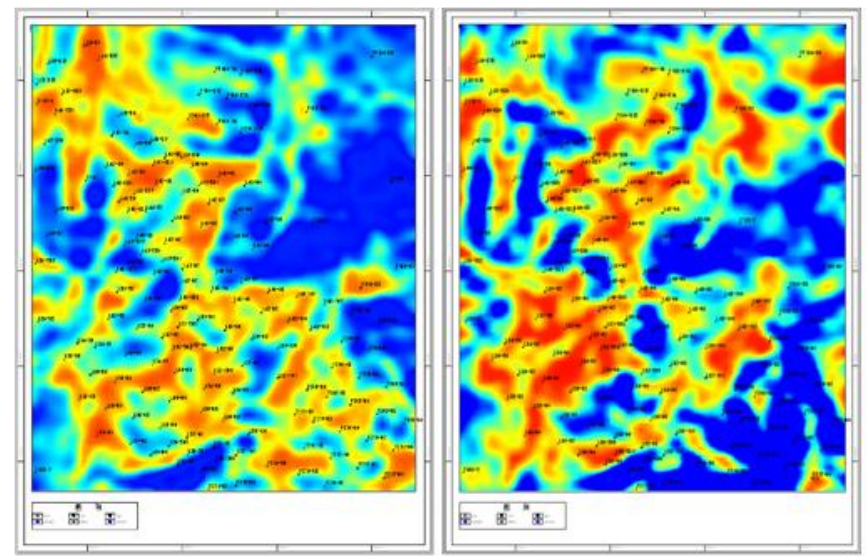

Figure1.4 seismic attribute plan of target layer

\subsection{Sand body prediction}

\section{Sand Body Prediction And Effect Analysis By Seismic Inversion}

After the optimization of the inversion parameters in the study area, it was necessary to start the whole work area inversion and carry out the sandstone prediction work ${ }^{[8-10]}$. Before the sandstone prediction, we first made a statistical analysis of sandstone data and wave impedance data in the well, and tried to establish the relationship between the wave impedance and the sandstone ${ }^{[11-12]}$. But from the view of figure 2.1, in addition to the P112 layer, the other layer wave impedance could not distinguish between sand and mudstone, so the use of the original curve to directly carry out geostatistical inversion could not solve the reservoir prediction problem in this area.
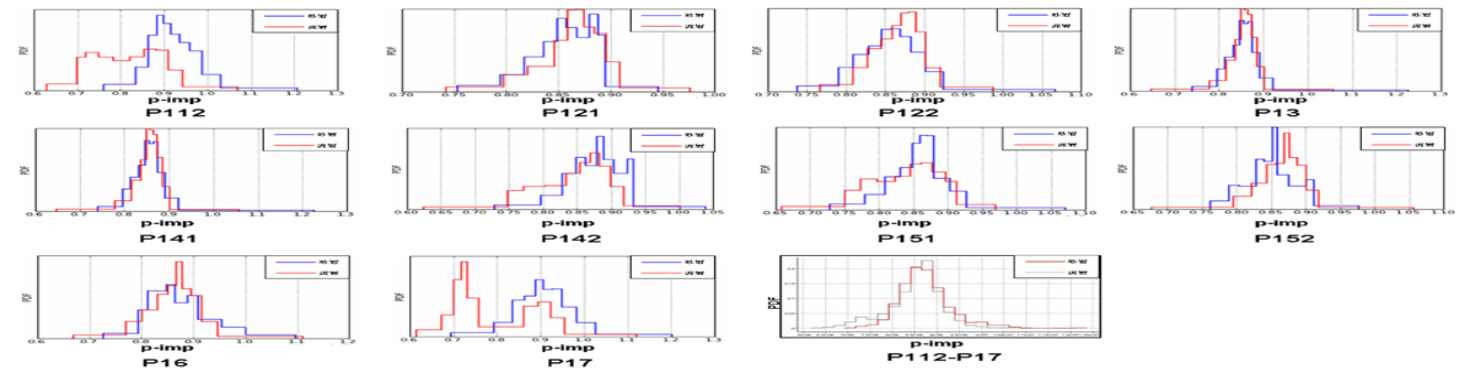

Figure2.1 Histogram of relationship between reservoir sand shale and wave impedance in Putaohua oil layer 
So we need to find the relationship with other curves and sand shale, in Figure 2.1, the GR curve and the sand mudstone was most closely related, so we applied the GR curve to fit the wave impedance, using pseudo wave impedance inversion to distinguish between sandstone and mudstone (Figure 2.2).

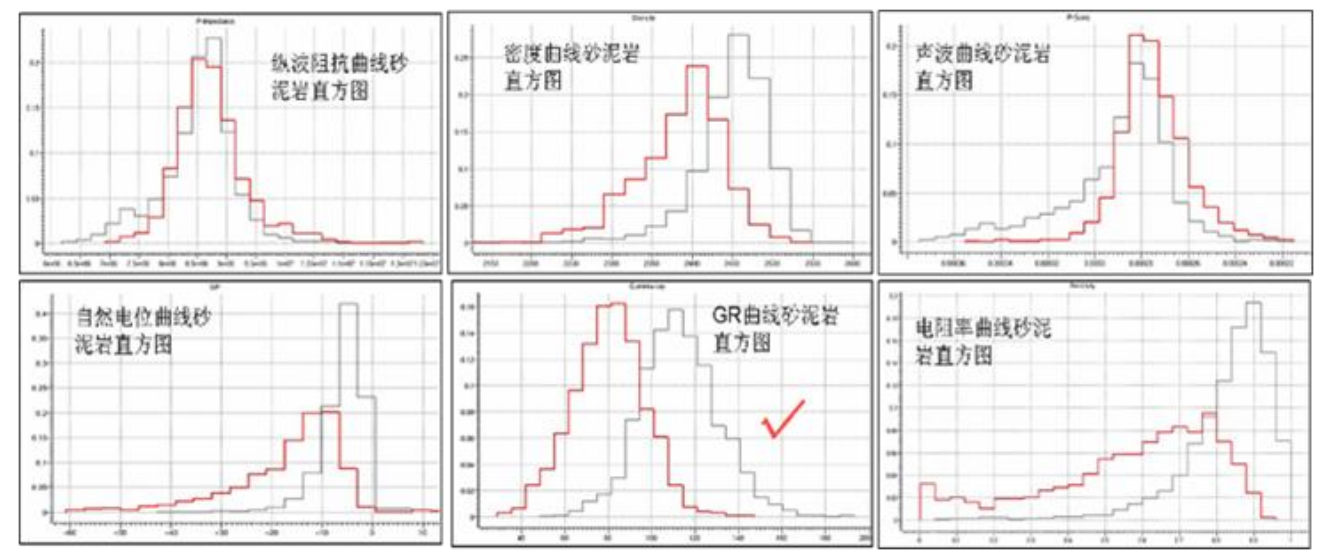

Figure2.2 Histogram of sand shale and logging curve

Sandstone prediction was carried out by pseudo impedance inversion and statistical inversion in the study area. This method combines the advantages of pseudo sonic and geostatistical inversion, which is to ensure the vertical resolution, and accurate identification of sand body. This paper adopted the latest acoustic inversion method, which corrected the shortcomings of conventional methods and had stronger ability to distinguish the lithology. Firstly, the combined inversion method of pseudo sonic and geostatistics was tested by using the Chang 48-S87- 204-90 well profile. The results showed that the dissonation of the constrained sparse pulse inversion was too low, the original wave impedance geostatistics inversion had a low coincidence rate, and the profile was not natural. However, the combined inversion method of pseudo sonic and geostatistics was more natural. The vertical resolution ability and lateral extension were better, and the coincidence rate of wells was higher.

\subsection{Effect analysis}

In order to verify the correctness of the inversion method and the inversion parameter, 48 wells were chosen as the posterior test well to verify the accuracy of the inversion results. The selection principle of the posterior well, (1) the well does not control the whole or part of the area; (2) when the well is close, draw the well at the separation point; (3) the result of the inversion is not good, and the number of the later inspection wells is reduced appropriately. From the inversion section, the longitudinal posterior well was in good agreement with the inversion results (Figure 2.3).

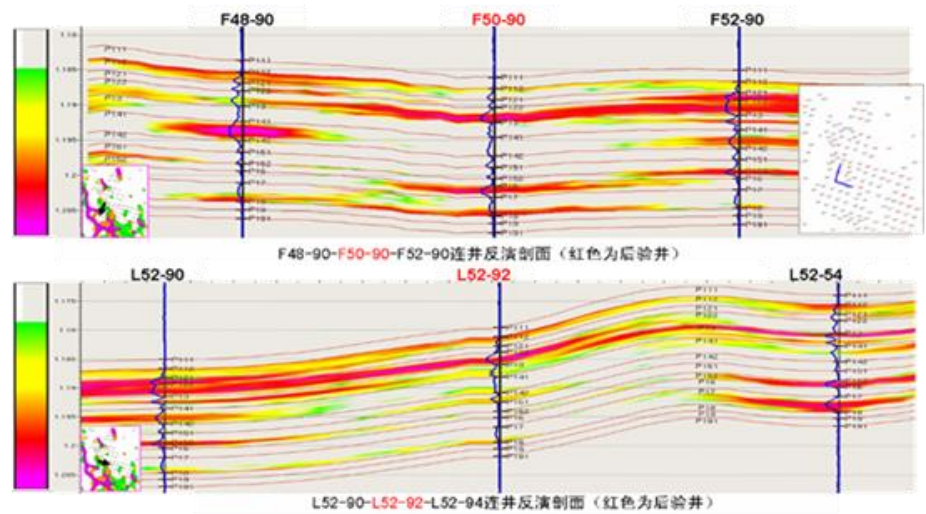

Figure 2.3 posterior (red) inversion section

From the view of continuous well line of Fang 48-90- Fang,50-90- and Fang 52-90. The well 50-90 had a sandstone development in the Putaohua I22 reservoir and the I6 oil layer, and corresponded well to the SP curve, which was basically consistent with the well curve in the vertical direction. In Yong 52-90- Yong 52-92Yong 52-94 continuous well line, the test well Yong 52-92 well corresponded to the well SP curve in the Putaohua I22 oil layer. However, the predicted sandstone development position was different from that of the SP curve in Putaohua I7 reservoir. In general, the inversion results agreed well with the posterior well. 
Look from the plane, with Portuguese 22 reservoir as an example, the inversion results also had good agreement with the development of Sandstone on the well, $2 \mathrm{~m}$ sand body coincidence rate was $78.3 \%, 1-2 \mathrm{~m}$ coincidence rate was $52.2 \%$, which can met the requirements of reservoir prediction in the study area. Using the inversion results of geological statistics, the inversion plan of each target layer in the study area (Figure 2.4) was extracted, combining well information and seismic attribute analysis, fine description and description of sedimentary facies of well seismic combination were carried out.

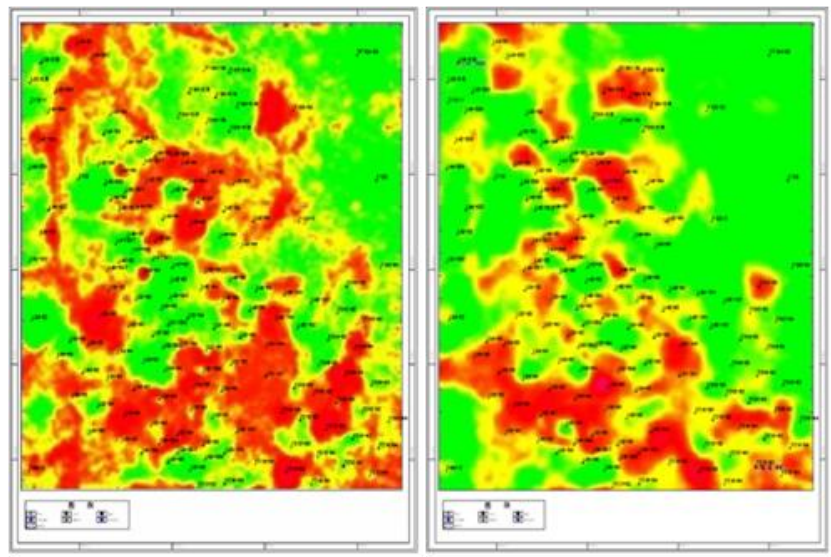

Figure 2.4 Inversion plane graph of target layer

\section{Conclusion}

1. In the process of thin sand body identification, the quality of seismic data is very important. In order to preserve more and more effective information in seismic data, we need to carry out high fidelity processing to meet the needs of the current thin sand prediction.

2. The combination of well information and seismic information can effectively identify thin channel sand bodies and sheeted sand bodies. Seismic information is effective in single channel identification, distributary channel distribution and connectivity, facies boundary determination and inter well prediction.

3. The three step combined with seismic facies description scheme is established. Through the combination of well and seismic data, the overall characteristics were taken into account, local details were corrected, a comprehensive analysis map was made by using well and seismic information. Compared with the original sedimentary facies map, the source direction and overall sedimentary distribution characteristics of the sedimentary unit of the oil reservoir group are grasped. Based on the analysis of the well and seismic information, the accuracy of the information in the well was emphasized, the lateral continuity of the seismic information was grasped, and the local details were corrected by the combination of the well and seismic. The comprehensive analysis showed that the continuity of channel sand in the study area was better, the branch of river was increasing, the single channel increased in the sedimentary unit, and the sand distribution between the wells was more precise.

\section{Reference}

[1]. Zheng Rongcai, Zhou Qi, Wang Hua, et al. High resolution sequence configuration and sand body prediction of the 2 section of Shanxi formation in Chang Bei gas field, Ordos Basin [J]. Journal of the Chinese University of geology, 2009, 15 (1): 69-79.

[2]. Sun Yu, Zhao Dan, Yu Limin, et al. Shallow lacustrine fluvial delta front sandbody distribution characteristics and sedimentary models -- Based on grape Yongle region in northern Songliao Basin of Putaohua reservoir as an example [J]. Acta sedimentologica Sinica, 2015, 33 (3): 439-447.

[3]. Sun Zuoxing, Zhang Yina, Liu Changli, et al. Sedimentary characteristics and significance of oil and gas exploration in shallow water delta [J]. petroleum and natural gas journal, 2012 (9): 161-165.

[4]. Lv Xiaoguang, Li Changshan, Cai Xiyuan, et al. Delta sedimentary characteristics and reservoir structure model of frontal reservoir in Songliao large shallow lake basin [J]. Journal of sedimentary science, 1999 (4): 572-577.

[5]. Sui Jun. study on fluvial delta reservoir in Daqing oilfield [M]. Petroleum Industry Press, 2000

[6]. Li Yuanhao, Liu Chiyang, Du Yu Guo, et al. Sedimentary characteristics of shallow water delta in the Yanchang Formation of three Yanchang Formation in the northwestern Ordos Basin and the sand controlling sand by the lake, [J]. palaeogeography, 2009, 11 (3): 265-274. ()

[7]. Yin Taiju, Li Xuanyue, Zhang Changmin, et al. Morphological characteristics of deltaic sedimentary sand bodies in modern shallow water lake basins -- Taking Dongting Lake and Poyang Lake as examples, [J]. petroleum and natural gas journal, 2012, 34 (10): 1-7.

[8]. Huang Zhenping, Wang Xiaohua, Wang Yun. Special parameter analysis and thickness prediction of thin layer seismic [J]. petroleum geophysical exploration, 1997 (3): 28-38

[9]. Wang Ying. Geophysical inversion theory, [M]., higher education press, 2002.

[10]. Yang Wen. Theory and method of geophysical inversion [M]. Geological Publishing House, 1997. land based, Meng. Principle of seismic exploration,.2[M]. Petroleum University Press, 1993.

[11]. Li Qingzhong. The road to precise exploration: analysis of high resolution seismic exploration system engineering, [M]. petroleum industry press, One thousand nine hundred and ninety-four 